\section{Wolfgang Raible}

\section{Medien-Kulturgeschichte}

Mediatisierung als Grundlage unserer kulturellen Entwicklung

Heidelberg: Univ.-Verl. Winter, 2006. - 461 S.

(Schriften der Philosophisch-historischen

Klasse der Heidelberger Akademie der Wissenschaften; 36)

\section{ISBN 3-8253-5170-X}

Wer eine Medien-Kulturgeschichte schreibt, hat eine ganze Reihe von grundsätzlichen Fragen zu beantworten: Inwiefern lassen sich mediale und kulturelle Prozesse beobachten und erklären? In welchem Verhältnis stehen Medien und Kultur? Welche Rolle spielt der Mensch? Wie lassen sich Medien-, Kultur- oder sogar Medienkulturgeschichten überhaupt schreiben?

Im Gegensatz zu antihermeneutischen Ansätzen mit technikdeterministischer Ausrichtung hält Wolfgang Raible mit seiner Medien-Kulturgeschichte am semiotischen Modell der Welterklärung fest. „Wer mehr weiß, kann auch mehr Zeichen als Zeichen erkennen." (S. 4), so lautet sein Credo. Die Generierung von Bedeutung wird als bewusster Akt der Selektion verstanden. Bedeutung entsteht einerseits durch Auswahl, andererseits durch das interpretierende Schließen von Lücken. Hierarchiebildung einerseits und die Einordnung der Zeichen innerhalb einer Zeichenhierarchie andererseits sind die Grundlagen von Raibles Verstehensmodell. Zentral für sein Verständnis der Weltwahrnehmung und Wissensverarbeitung ist die Rolle des Menschen als sinngebender Instanz (vgl. S. 7).

In Hinblick auf die Frage nach dem Abhängigkeitsverhältnis von Medien und Kultur vertritt Raible eine explizit anthropologische Position. Er versucht, die Mediengeschichte konsequent vom Anwender bzw. von den symbolischen Formen des Gebrauchs her zu denken. Bei der Marginalisierung der Medientechnologien - wie Buchdruck oder Internet - geht er manchmal sogar so weit, dass man die Rolle der Medien im Prozess der Mediatisierung fast aus den Augen verliert. Die Unsichtbarkeit der Medien, die im Kontext unsichtbarer digitaler Codierungsprozesse $\mathrm{zu}$ einer zunehmenden Erblindung medienbezogener Theoriebildung zu führen droht, ist auch bei Raible Programm. Woher sich die Schwierigkeiten der Beobach- tung von Medien und Kulturen ableiten oder gar welche erkenntnistheoretischen Entwicklungen zu diesem gegenwärtigen Problemstand geführt haben, darüber erfährt man in Raibles Band nur wenig.

Anknüpfend an das Geschichtsmodell Fernand Braudels charakterisiert er die Mediengeschichte als emergenten Prozess, in dem vor allem mittel- und langfristige Perspektiven von Bedeutung sind (vgl. S. 50-52). Den Modellen einer teleologischen Geschichtsschreibung, die Geschichte als lineare, zielgerichtete Abfolge von erfolgreichen Erfindungen und Erfindern präsentieren, erteilt Raible ebenso eine Absage wie der historischen Kurzsichtigkeit einer faktenorientierten Ereignisgeschichte, die Mediengeschichte auf eine chronologische Abfolge einzelner medienhistorischer Sternstunden reduziert. Größere Sympathien hegt er für die Medienarchäologie, mit deren Hilfe Raible einerseits die Brüche und Sackgassen in der Entwicklung der Medien aufzudecken hofft, die ihn andererseits jedoch zu einer nicht immer ganz nachvollziehbaren Auswahl an Themen, Epochen und Entwicklungen verführt.

So aufschlussreich Raibles Band in Hinblick auf die Profilierung der Mediengeschichte als Kultur- und Wissenschaftsgeschichte ist, so wenig eignet er sich zur medienkulturhistorischen Orientierung. Wer sich von dem Band einen systematischen Überblick über die Medien-Kulturgeschichte von ihren Anfängen bis heute erwartet, wird enttäuscht. Mag sein, dass dies auch gar nicht das Ziel Raibles war. Selbst wenn der Klappentext vollmundig ankündigt, dass der „Verfasser [...] den Begriff ,Medien’ in seiner ganzen historischen Tiefe" entfalte, so mag dies wohl vor allem für den eigentlichen Geltungsbereich des Philologen und Sprachwissenschaftlers Raible, weniger aber für die aktuelleren Arbeitsfelder der Medien- und Kommunikationswissenschaften gelten.

Ihre medienkulturhistorischen Stärken hat die Studie in den Bereichen der Schrift- und Printgeschichte, wobei Raible hier bekanntermaßen zu den Kritikern jener Ansätze zählt, die der Erfindung des Buchdrucks durch Gutenberg eine epochale Bedeutung zuschreiben. Die zentralen Entwicklungen für den kulturhistorischen Siegeszug der Schrift verortet Raible in einer Reihe von Leseerleichterungen, wie sie schon um 1200 im Kontext der Scholastik eingeführt wurden. Den Brückenschlag $\mathrm{zu}$ aktuellen Medienentwicklungen vollzieht 
der Philologe Raible am Beispiel des Internets, einer Technologie, die aufgrund ihrer digitalen Codierung eine große Affinität zur Geschichte alphanumerischer Aufschreibesysteme aufweist. In diesem Kontext hat der Band auch einen seiner inhaltlichen Ausgangspunkte: einen Vortrag über Genese und Funktion des Internets. Darin entwickelte Raible „den Gedanken, das Internet als Resultat einer Entwicklung der longue durée und, vor allem, als ein Instrument von Wissenschaftlern für Wissenschaftler zu sehen" (S. 400). Aus dem Blickwinkel der symbolischen Formen, die Raible über die Bedeutung der Medientechniken stellt, hat das Internet seine Wurzeln nicht in der Hardware militärischer Technokratie, sondern im humanistischen Projekt einer enzyklopädischen Erfassung der Welt. Nicht das Internet als technologischer Rahmen, sondern Online-Enzyklopädien wie Wikipedia sind das Telos digitaler Medienentwicklung.

Mit seiner demonstrativen Bagatellisierung von Medientechnologien wie Buchdruck oder Internet umgeht Raible zwar den technischen Determinismus der Medien, in seiner kulturhistorischen Argumentation konstruiert er insgeheim aber dennoch wieder teleologische Geschichten der Mediennutzung, die sich manchmal mehr, manchmal weniger nachvollziehen lassen, im Einzelnen aber oft nur schwer zu belegen sind.

Eher mager ist auch die informative Ausbeute in den Kontexten von Film, Hörfunk oder Fernsehen. Eine Darstellung der Filmgeschichte aus dem Geist des Internet Movie Database (IMDb) mag zwar medienarchäologischen Interessen der Zukunft vorausgreifen, als Einführung oder Überblick zum Thema wirkt sie eher kurios (vgl. S. 336-362). Ähnliches gilt für den Hörfunk und das Fernsehen. Das Thema Hörspiel wird ein Mal mehr mit einigen allseits bekannten Zitaten aus Orson Welles' War of the Worlds abgehandelt (vgl. S. 249-252). Im Hinblick auf die neuen symbolischen Formen des Fernsehens beschränkt sich Raible im Wesentlichen auf eine Kurzdarstellung der in der Tradition von roman fevilleton und radioteatro stehenden Telenovela (vgl. S. 270-275). Die Vielfalt an technischen, kulturellen, ökonomischen und juristischen Informationen entspricht zwar dem multiperspektivischen Ansatz der Medienkulturwissenschaften, bleibt im vorliegenden Fall aber häufig auf recht oberflächlichem Niveau.
Formal kann Raibles Medien-Kulturgeschichte die medialen Grundlagen ihrer Entstehung - die Transformation einer Power-PointVorlesung in einen ausformulierten Text - nicht verleugnen. Was als Vorlesung mit multimedialer Unterstützung begeistert, kann als Buch nicht wirklich überzeugen. Die schlampige Form, häufig fehlende Fußnoten und Quellenangaben tragen das Ihre dazu bei, dass das erklärte Ziel einer transparenten Mediengeschichte als Kultur- und Wissenschaftsgeschichte leider nur ansatzweise erreicht wird.

Heinz Hiebler

\section{Gabriele Siegert / Rolf H. Weber / Frank Lobigs / Dirk Spacek}

\section{Der Schutz innovativer publizistischer Konzepte im Medienwettbewerb}

Eine medienökonomische und medienrechtliche Untersuchung

Baden-Baden: Nomos, 2006. - 157 S.

(Schriften zur Medienwirtschaft und zum Medienmanagement; 15)

ISBN 3-8329-2394-2

Der Konzeptschutz ist eines der großen ungelösten Probleme des Urheberrechts. Das Urheberrecht schützt Ideen nicht und überträgt diesen Grundsatz der Ideenfreiheit auch auf innovative publizistische Konzepte, wie z. B. Fernsehformate, Zeitschriftenkonzepte oder Werbekonzeptionen. Nachdem in verschiedenen europäischen Staaten, etwa in Deutschland durch den Bundesgerichtshof, der Schutz von Formaten definitiv abgelehnt worden ist, mehren sich Stimmen, die eine Neuausrichtung des Immaterialgüterrechts fordern. Erwähnt sei hier z. B. die in Köln ansässige Format Recognition and Protection Association (Frapa), ein internationaler Lobbyverband für den Schutz von Fernsehformaten. Das starke Engagement der Frapa ist jedoch wissenschaftlich noch nicht analysiert und hinterfragt worden. Insofern stoßen die Autoren des vorliegenden Bandes auf eine Forschungslücke, als sie im Jahre 2005 mit Mitteln des schweizerischen Nationalfonds ein Forschungsprojekt zur Klärung dieser schwierigen Fragen ins Leben gerufen haben. Die vorliegende Untersuchung skizziert insofern auch die Endergebnisse dieses Forschungsprojektes, das sehr innovativ ökonomische Ansätze mit immaterialgüterrechtlichen Fragestellungen verbindet. 